\title{
MR Imaging in Patients with Cardiac Pacemakers and Implantable Cardioverter Defibrillators*
}

\author{
Consensus Paper of the German Cardiac Society and the \\ German Roentgen Society
}

\section{MR-Untersuchungen bei Patienten mit Herzschrittmachern und implantierbaren Kardioverter-Defibrillatoren}

\author{
Konsensuspapier der Deutschen Gesellschaft für Kardiologie (DGK) \\ und der Deutschen Röntgengesellschaft (DRG)
}

\section{Authors}

Torsten Sommer ${ }^{1}$, Wolfgang Bauer ${ }^{2}$, Katharina Fischbach ${ }^{3}$, Christof Kolb ${ }^{4}$, Roger Luechinger ${ }^{5}$, Uwe Wiegand ${ }^{6}$, Joachim Lotz ${ }^{7}$, Ingo Eitel ${ }^{8}$, Matthias Gutberlet ${ }^{9}$, Holger Thiele ${ }^{10}$, Hans H. Schild ${ }^{11}$, Malte Kelm ${ }^{12}$, Harald H. Quick ${ }^{13}$, Jeanette Schulz-Menger ${ }^{14}$, Jörg Barkhausen ${ }^{15}$, Dietmar Bänsch ${ }^{16}$

Affiliations

1 Klinik für Diagnostische und Interventionelle Radiologie, DRK Krankenhaus Neuwied - Department of Diagnostic and Interventional Radiology, German Red Cross Hospital Neuwied, Germany

2 Med. Klinik und Poliklinik I Univ. Klinikum Würzburg, Deutsches Zentrum für Herzinsuffizienz Univ. Würzburg - Department of Internal Medicine I, University Hospital Würzburg, Germany, Center for Heart Failure Research, University of Würzburg, Germany

3 Klinik für Radiologie und Nuklearmedizin, Universitätsklinikum Magdeburg - Department of Radiology and Nuclearmedicine, University Hospital Magdeburg, Germany

4 Klinik für Herz- und Kreislauferkrankungen, Deutsche Herzzentrum Muenchen, Muenchen, Deutschland- Department of Cardiology, German Heart Center Munich, Germany

5 Institut für Biomedizinische Technik, Universität und ETH Zürich Institute for Biomedical Engineering, University and ETH Zurich, Switzerland

6 Klinik für Kardiologie, Schwerpunkt Rhythmologie und Elektrophysiologie, Sana-Klinikum Remscheid, Remscheid Department of Cardiology,Rhytmology and Electrophysiology, Sana-Clinics Remscheid, Remscheid, Germany

7 Diagnostische und Interventionelle Radiologie, Universitätsmedizin Göttingen - Diagnostic and Interventional Radiology, University Hospital Göttingen, Germany

8 Medizinische Klinik II (Kardiologie, Angiologie, Intensivmedizin), Universitäres Herzzentrum Lübeck, Universitätsklinikum Schleswig-Holstein (UKSH) - Department of Internal Medicine II (Cardiology, Angiology, Intensive Care) University Heart Center Luebeck, University Hospital, Schleswig-Holstein, Germany

9 Department of Diagnostic and Interventional Radiology, University of Leipzig - German Heart Center Leipzig, Germany
10 Medizinische Klinik II (Kardiologie, Angiologie, Intensivmedizin), Universitäres Herzzentrum Lübeck, Universitätsklinikum Schleswig-Holstein (UKSH) - Department of Internal Medicine II (Cardiology, Angiology, Intensive Care) University Heart Center Luebeck, University Hospital, Schleswig-Holstein, Germany

11 Radiologische Klinik, Unversitätsklinikum Bonn - Department of Radiology, University Hospital Bonn, Germany

12 Klinik für Kardiologie, Pneumologie und Angiologie, Universitaetsklinikum Duesseldorf, Duesseldorf -Department of Cardiology, Pneumolgy and Angiology, University Hospital Duesseldorf, Duesseldorf, Germany

13 Hochfeld- und Hybride MR-Bildgebung, Universitätsklinikum Essen, Essen; Erwin L. Hahn Institut für MR-Bildgebung, Universität Duisburg-Essen, Essen - High Field and Hybrid MR Imaging, University Hospital Essen; Erwin L. Hahn Institute for MR Imaging, Germany

14 Experimental Clinical Research Center, Kardiologie, Charité Campus Buch, ECRC, Universitätsmedizin Berlin, Berlin, Deutschland, HELIOS Klinikum Berlin-Buch, Klinik für Kardiologie und Nephrologie, Deutschland - Experimental Clinical Research Center,Cardiology, Charité Campus Buch, University Medicine Berlin Germany, HELIOS Clinics Berlin-Buch, Department Cardiology and Nephrology, Berlin, Germany

15 Klinik für Radiologie und Nuklearmedizin, Universitätsklinikum Schleswig-Holstein - Department of Radiology and Nuclearmedicine, University Hospital Luebeck, Germany

16 Klinik für Innere Medizin/Kardiologie KMG-Kliniken Güstrow, Deutschland - Department of Internal Medicine/Kardiology, KMG Hospital Guestrow, Germany

Key words MRI, pacemaker, ICD, guideline, MRI safety

received 8.1.2017

accepted 31.1.2017

Bibliography

DOI http://dx.doi.org/10.1055/s-0043-102029

Published online: 15.2.2017 | Fortschr Röntgenstr 2017; 189: 204-

217 (c) Georg Thieme Verlag KG Stuttgart · New York

ISSN 1438-9029

* The German language version of this article is simultaneously published in issue 2/2017 of "Der Kardiologe". 
Correspondence

Priv.-Doz. Dr. Torsten Sommer

Department of Diagnostic and Interventional Radiology,

German Red Cross Hospital Neuwied

Marktstr. 104

D- 56564 Neuwied

Germany

Tel.: ++49/2631/981751

Fax: ++49/2631/98 1790

torsten.sommer@drk-kh-neuwied.de

\section{ABSTRACT}

This joint consensus paper of the German Roentgen Society and the German Cardiac Society provides physical and electrophysiological background information and specific recommendations for the procedural management of patients with cardiac pacemakers (PM) and implantable cardioverter defibrillators (ICD) undergoing magnetic resonance (MR) imaging. The paper outlines the responsibilities of radiologists and cardiologists regarding patient education, indications, and monitoring with modification of MR sequences and PM/ ICD reprogramming strategies being discussed in particular. The aim is to optimize patient safety and to improve legal clarity in order to facilitate the access of SM/ICD patients to MR imaging.

\section{Key Points:}

- Conventional PM and ICD systems are no longer an absolute but rather a relative contraindication for performing an MR examination. Procedural management includes the assessment of the individual risk/benefit ratio, comprehensive patient informed consent about specific risks and "off label" use, extensive PM/ICD-related and MR-related safety precautions to reduce these risks to the greatest extent possible, as well as adequate monitoring techniques.
- MR conditional pacemaker and ICD systems have been tested and approved for MR examination under specific conditions ("in-label” use). Precise understanding of and compliance with the terms of use for the specific pacemaker system are essential for patient safety.

- The risk for an ICD patient during MR examinations is to be considered significantly higher compared to PM patients due to the higher vulnerability of the structurally damaged myocardium and the higher risk of irreversible damage to conventional ICD systems. The indication for a MR examination of an ICD patient should therefore be determined on a stricter basis and the expected risk/ benefit ratio should be critically reviewed.

- This complex subject requires close collaboration between radiology and cardiology.

\section{Citation Format}

- Sommer T, Bauer W, Fischbach K et al. MR Imaging in Patients with Cardiac Pacemakers and Implantable Cardioverter Defibrillators. Fortschr Röntgenstr 2017; 189: 204-217

\section{ZUSAMMENFASSUNG}

In diesem gemeinsamen Konsensuspapier der Deutschen Röntgengesellschaft und der Deutschen Gesellschaft für Kardiologie werden der MR-physikalische und elektrophysiologische Hintergrund sowie das prozedurale Management für MR-Untersuchungen von Patienten mit Herzschrittmachern und implantierbaren Kardioverter-Defibrillatoren dargestellt. Es werden detaillierte Handlungsanweisungen bezüglich Indikationsstellung, den spezifischen Untersuchungsbedingungen mit Umprogrammierung des SM/ICD-Systems, Modifikation der MR-Sequenzen und Monitoring gegeben sowie die Verantwortlichkeiten und Aufklärungsnotwendigkeiten zwischen Radiologie und Kardiologie definiert. Ziel ist es, ein Maximum an Patientensicherheit zu gewährleisten sowie Rechtssicherheit für die beteiligten Ärzte herzustellen, um insgesamt den Zugang von Patienten mit aktiven kardialen Implantaten zu notwendigen MR-Untersuchungen zu verbessern.

\section{Introduction}

The presence of a pacemaker (PM) or implantable cardioverter defibrillator (ICD) system has long been considered an absolute contraindication for MR imaging (MRI). However, various studies in the last 15 years have been able to show that it is possible to perform MR examinations with an acceptable level of risk in patients with conventional PM/ICD systems taking into consideration the individual risk/benefit profile as a single-case decision and as "off-label" use and applying specific safety precautions [1 - 20]. In 2008, a European position paper regarding this topic with participation of corresponding expert groups from cardiology and radiology was published and the presence of PM/ICD systems was newly classified as a relative MR contraindication [1].

In the same year MR conditional or MR compatible pacemaker systems were introduced for the first time and are now offered by all major pacemaker manufacturers. These systems - defined as a functional unit composed of the pacemaker pulse generator, leads, and a software-based MR protection mode - are tested and approved for MR examination under certain conditions.

Experience in recent years in Germany has shown that use of the systems varies greatly. While some specialized centers perform MR examinations as an off-label procedure even in patients with conventional PM/ICD systems with a corresponding indication under the recommended specific safety precautions, many MR facilities refuse to investigate patients with pacemakers or ICDs, even those with MR conditional systems. One reason for this is a significant lack of information and legal uncertainty on the part of both radiology and cardiology with respect to the concrete handling of these new developments and this technically complex and interdisciplinary issue in the clinical setting.

Updated recommendations/guidelines regarding this topic were published in 2013 by the European Society of Cardiology (ESC) [21] and in 2015 by the German Roentgen Society [22] but relate, particularly in the ESC publication, to the own specialty and do not provide a procedure that has been coordinated and approved by both societies.

This joint consensus paper of the German Roentgen Society and the German Cardiac Society provides physical and electrophysiological background information and specific recommendations for the procedural management of patients with PMs and ICDs, outlining the responsibilities of radiology and cardiology regarding patient education, indications, and monitoring with PM/ICD reprogramming being discussed in particular.

The following recommendations relate to the total clinical spectrum of MR examinations. Cardiac MR, which represents 
only a relatively small percentage of the total number of MR examinations but is becoming increasingly important in the diagnosis of cardiac diseases, is discussed separately.

\section{MR imaging in patients with active cardiac implants - physical and electrophysiologi- cal background}

There can be interactions between active cardiac implants (i. e., PMs and ICDs) and MR scanners that can be attributed to an interaction with the electromagnetic fields in the MR system, i. e., the static magnetic field $\left(B_{0}\right)$, the gradient fields $\left(G_{x}, G_{y}, G_{z}\right)$, and the high-frequency fields $\left(B_{1}\right)$.

The static magnetic field has a field strength of $0.5-3.0$ Tesla in most clinical MR systems and is necessary to polarize the spins of hydrogen protons and thus to achieve nuclear magnetization. This strong magnetic field affects the ferromagnetic components of the implant. This results in an attractive force that is at its maximum in the immediate vicinity of the MR system in the region of the opening of the MR tunnel as well as a torque that is at its maximum in the center of the MR tunnel. These forces can result in paresthesia in individual cases in the region of the pacemaker pocket in older pacemaker models but are not large enough to dislocate ingrown PM/ICD systems or leads. In modern active cardiac implants, the ferromagnetic components have been largely reduced so that the effective forces have been decreased by a factor of approximately 10 and are lower than the gravitational forces acting on them [8].

Older pacemakers and ICDs use a mechanical switch, known as the reed switch, as a control element. These reed switches are activated (closed) by a weak magnetic field in the form of a hand magnet, resulting in a mode switch to the asynchronous stimulation mode (D00 or V00) with deactivation of the sensing function in pacemakers and in deactivation of tachycardia detection in ICDs. However, these reed switches do not function properly in the strong static magnetic fields of MR systems. Since the torque effect predominates here, $50 \%$ of switches remain deactivated (open) under consideration of all possible spatial orientations [9]. Since the behavior of the reed switch in the individual case cannot be predicted, there is a potential safety risk during an MR examination, e.g. due to uncontrolled asynchronous stimulation. In current devices and in particular in all MR conditional devices, Hall sensors are used instead of mechanical reed switches as the magnetic field detectors which function properly even in strong static magnetic fields.

Movement in the static magnetic field as well as voltages induced by the gradient and high-frequency fields can result in an electrical reset in older conventional devices with activation of an emergency mode (typically VVI). This can result in suboptimal pacing since individual stimulation parameters or protective antitachycardiac functions will be reset. According to current data, these electrical resets, which represent a potential safety risk, no longer occur in MR conditional pacemakers due to modifications and improved protection of the internal circuits.

The gradient fields are active during MR imaging to achieve spatial encoding of the MR signal. They have maximum slopes up to $80 \mathrm{mT} / \mathrm{m}$ and can be activated and deactivated in the submillisecond range (slew rate up to $200 \mathrm{~T} / \mathrm{m} / \mathrm{s}$ ). The gradient fields are therefore temporally variable electromagnetic fields and can induce voltage pulses in the leads. This can be perceived by the $\mathrm{PM} / \mathrm{ICD}$ system as intrinsic action of the heart with consecutive inhibition of PM stimulation or in the worst case can lead to a stimulation pulse with asynchronous myocardium stimulation. According to current data, the risk of cardiac stimulation is largely ruled out in the case of MR conditional pacemakers as a result of modifications to the electrical filters and protective circuits in the system.

The most complex and most significant interactions occur between the implant and the radiofrequency field of the MR unit. The radiofrequency field is necessary to excite the hydrogen protons to generate the MR signal. The frequency of these field referred to as high frequency (HF) or radio frequency (RF) depends on the magnetic flow density based on the gyromagnetic ratio and has magnetic field strengths of approximately $64 \mathrm{MHz}$ at 1.5 Tesla respectively $128 \mathrm{MHz}$ at 3.0 Tesla. These RF fields are electromagnetic waves and therefore possess in addition to the magnetic field an electrical field component that can interact with the conductive lead of the implant. The wave length of the RF field in the human body is approximately $50 \mathrm{~cm}$ at 1.5 Tesla and $25 \mathrm{~cm}$ at 3.0 Tesla due to the high dielectricity constant of human tissue. If PM or ICD leads have a length of whole or half-integral multiples of the wave length of the RF field, resonance effects can result in particularly high energy deposition at the lead tips. Like an antenna, the lead can receive the RF signal, locally intensify it and transmit its energy to the lead tip and from there to the heart muscle tissue resulting in thermal tissue damage and irreversible pacing capture threshold increases. The level of energy absorption and transmission to the tissue depends on many factors, such as the position and path of the lead in the patient, the position of the patient in the MR system, the design and insulation of the lead and the electrical design of the RF transmitter coil used in the system. In phantom experiments with clinically realistic system-lead configurations as well as in animal experiments, it was shown that the RF field can induce a significant temperature increase ( $\Delta \mathrm{T}$ up to $65^{\circ} \mathrm{C}$ ) at the lead tip with the leads in certain positions [7, 10, 20]. In addition, it was shown that the coupling of a pacemaker pulse generator to the lead tends to reduce the RF-induced heating of the electrode tip. This is important, for example, when implanted leads must be disconnected (abandoned leads), which further increases the risk of potential heating of the leads.

Although the frequency of the RF field is much too high to result in direct depolarization of the cardiac muscle cell and thus cardiac contraction, rectification processes, e. g. via the nonlinear response of a protective diode in the system, are fundamentally possible. This may result in cardiac stimulation and the induction of ventricular tachycardia. Moreover, the RF field during these rectification processes - similarly to gradient fields - can result in inhibition of the pacemaker and erroneous tachycardia therapy. 


\section{Incidence of complications of MR imaging in pacemaker and ICD patients}

The real incidence of complications in MR examinations of patients with PM or ICD systems cannot be determined from real world data or register data since MR examinations in these patients were absolutely contraindicated until recently. There are only individual reports of complications from case reports of patients who underwent an MR examination without specific safety precautions because the physician was unaware of the presence of a pacemaker/ICD system. This includes at least 7 cases with a fatal outcome [28]. Both the true number of MR-related complications and the reference value, namely the total number of patients who underwent such an "accidental" MR examination, are not known.

Alternatively, it is possible to use the controlled studies in which PM/ICD patients underwent an MR examination under dedicated safety precautions and after detailed review of pacemaker function.

Under these controlled conditions, clinically relevant complications (bradyarrhythmia or tachyarrhythmia requiring treatment, lead dysfunction with the need for lead revision, damage to components or the total integrity of the pacemaker with the need to replace the system, myocardial infarction, pericardium perforation, death) were not reported in any of these studies with a cumulative total number of over 1000 patients.

However, various MR-induced effects that did not endanger patient safety were observed. The following should be mentioned here:

Before and after MR examination, statistically significant but very small and clinically irrelevant changes in the pacing capture threshold, impedance, and battery voltage [2] could be observed in pacemaker patients and lengthening of the charging time and a decrease in battery voltage [26] were seen in ICD patients. A small $(<20 \%)$ change in amplitude in the right atrium and right ventricle was observed in approximately $80 \%$ and $90 \%$ of device patients, respectively.

With respect to MR conditional PM and ICD systems, there are multiple prospective randomized clinical studies about the MR safety of these active cardiac devices. Clinically relevant MR-related events or complications have not been reported in any of these studies [29-32].

\section{MR imaging examinations in patients with conventional pacemaker systems \\ Critical review of indications for MR imaging in the case of conventional pacemakers}

The clinical urgency and therapeutic consequences of a requested MR examination must be documented by the referring clinical physician, and the lack of adequate imaging alternatives should be indicated by the attending radiologist. An interdisciplinary decision regarding the performance or withholding of the examination should be made on a case-by-case basis by the referring physician, cardiologist and radiologist.
Critical for this decision is the estimation of the individual risk/ benefit ratio taking into account the electrophysiological risk profile ( $\vee$ Table 1 ), the specific risk of the respective region to be examined ( $\vee$ Table 2 ), as well as local circumstances (expertise of the attending radiological and cardiological personnel, guarantee of qualified patient monitoring during the MR examination, as well as adequate management of any possible emergency situation).

Of the electrophysiological risk parameters illustrated in - Table 1, the first three items - 1. pacemaker dependency, 2. vulnerable myocardium/myocardial texture alterations with increased disposition for high-grade ventricular arrhythmias, as well as 3. abandoned pacemaker leads - should be emphasized as main risks during an MR examination of a patient with a conventional pacemaker system. In these cases the indication must be determined on a particularly strict basis. However, none of these three points necessarily implies withholding of an MR examination if - taking into account the expected clinical benefit and the specific risk for the region to undergo MR examination the risk/benefit ratio is considered positive for the patient on the whole [22].

\section{Patient information/informed consent in the case of conventional pacemakers}

During an appropriate time frame prior to MR examination, the patient should be specifically informed regarding the following circumstances and risks [22]:

1. MR examination of a patient with a conventional pacemaker is a non-approved ("off-label") procedure based on a case-bycase decision on the part of the attending physicians. The relevant authorities have not issued approval for MR examination, and neither pacemaker manufacturers nor the manufacturers of MR equipment will be liable for any damage or complications.

2. MR examination of a patient with a conventional pacemaker system poses the following potential risks and complications:

- Damage to the pacemaker system with the necessity of replacement

- Malfunction of the pacemaker leads, e.g. due to a heatingrelated irreversible increase of pacing capture thresholds, necessitating revision/extraction of the leads

- Heating of the pacemaker leads resulting in acute or chronic thermal damage to the cardiac muscle and ineffective pacemaker stimulation as a potentially life-threatening complication in the case of absolute PM dependency of the patient

- Induction of potentially life-threatening ventricular tachyarrhythmias

- Inhibition of pacemaker therapy of spontaneous bradyarrhythmic episodes occurring during MR examination with potentially life-threatening consequences in patients with absolute PM dependency

Occurrence of the risks described above can be minimized by employing specific safety precautions. However, these risks cannot be absolutely eliminated and are not quantifiable in individual cases. 
- Table 1 High-risk electrophysiological parameters for MR imaging of PM patients (modified according to [22]).

\section{electrophysiological parameters}

pacemaker dependence of the patient

vulnerable myocardium (acute/subacute myocardial infarction, acute myocarditis), arrhythmogenic substrate/structural cardiac disease

abandoned pacemaker leads/lead fragments

additional leads (e.g. coronary sinus/epicardial leads)

lead extensions, lead adapters

lead defect/fracture

metallic cardiac or extracardiac implants (length $>5 \mathrm{~cm}$ ) in direct vincinity $(<4 \mathrm{~cm})$ of the leads

primarily increased pacing capture threshold

low battery voltage (ERI, EOL criteria)

implantation time $<6$ weeks

- Table 2 Risk assessment of the MR examination region in patients with pacemakers undergoing MR imaging (modified according to [22]).

\section{increased potential risks}

- insufficient stimulation due to RF-induced tissue heating at the tips of the leads with increase of the pacing capture thresholds

- inhibition of stimulation due to gradient fields (false sensing)

elevated risk for induction of ventricular fibrillation/ventricular tachycardia via voltage induction in the leads due to gradient fields or rectified RF fields

elevated risk of RF-induced tissue heating at the tips of abandoned leads compared to leads connected to the pacemaker system

elevated/unknown risk of RF-induced heating effects at the lead tips due to the addition of individual antenna effects and/or elongation of the effective antenna length of the leads

elevated risk of RF-induced heating effects at the lead tip or site of lead fracture

elevated risk of RF-induced heating effects at lead tips due to the addition of the individual antenna effects

insufficient stimulation due to RF-induced tissue heating at the tips of the leads with further increase of the pacing capture threshold

elevated risk of electrical reset

unstable pacing capture thresholds in the healing phase after lead implantation; lead dislocation extremely improbable, but insufficient data available in early implantation period risk assessment of MR examination region

\section{higher risk}

thoracic spine, heart/chest, breast, shoulder (upper arm and elbow depending on the specific position)

\section{lower risk}

brain, pelvis, hip joint, knee, ankle/foot rationale

leads located completely in RF transmitter coil with increased coupling of RF energy and potentially increased heating effects at the lead

leads outside/largely outside the RF transmitter coil with limited RF energy coupling and only minimal heating effects at the lead tips

\section{Reprogramming strategies for conventional pacemakers}

The following possible interactions between the PM system and electromagnetic fields of the MR system must be taken into consideration in the reprogramming strategy for conventional PM systems:

1. Pulsed MR fields can result in complete inhibition of the PM with a corresponding potential risk for patients with absolute PM dependency.

2. In older PM models with a reed switch, activation of the reed switch (probability $50 \%$ ) by the static magnetic field results in asynchronous stimulation with the PM model-specific stimulation frequency (manufacturer-dependent between 85 and $100 / \mathrm{min})$. This can result in competitive rhythms with a po- tentially arrhythmic effect in patients with high intrinsic cardiac frequencies.

3. The electromagnetic interference induced by the MR fields and perceived by the PM can result in the PM no longer being able to discriminate between electromagnetic interference and intrinsic cardiac activity and thus switching to a protective mode with temporary asynchronous stimulation (so-called interference mode). Analogously to point 2, there is also a potential risk here for competitive rhythms.

4. A drop in battery voltage induced by the alternating electromagnetic fields of MR can result in a switch to emergency mode (electrical reset) with standard VVI programming. There is a potential risk when the original programming of V00 / D00 switches to VVI in patients with absolute PM dependency and the PM can thus be completely inhibited by the pulsed MR fields. 
In light of this, the following procedure is recommended as an expansion of the ESC Guidelines on Cardiac Pacing and Cardiac Resynchronisation Therapy published in 2013 [21], including practical instructions for the collaboration between attending cardiologists and radiologists:

a) In patients with absolute PM dependency (defined as intrinsic heart rhythm $<30 / \mathrm{min}$ ) and in patients without absolute PM dependency with permanent symptomatic bradycardia (intrinsic heart rhythm $<50 / \mathrm{min}$, individual deviations possible depending on symptoms and evaluation of the cardiologist), an asynchronous mode (D00 or V00) should be programmed significantly above the intrinsic heart frequency of the patient. The reprogramming before and after the MR examination should ideally be performed as close in time to the MR examination as possible. For feasibility and logistical reasons and depending on the individual evaluation of the attending cardiologist, initial PM reprogramming before the MR examination can be performed at a different time and location than the MR examination ( $\triangleright$ Table 3 ). Reprogramming of the PM with restoration of the original mode and a check of PM functionality and pacing capture thresholds must be performed immediately after the MR examination.

b) In non-PM-dependent patients with higher or unstable intrinsic heart rates there are 2 options for reprogramming:

1. Deactivation of the stimulation function of the PM

The stimulation function of the PM is deactivated by reprogramming to 0D0, 000, or, if both options are not possible in the case of older PM models, the stimulation output is reprogrammed to "subthreshold". From an electrophysiological standpoint, this procedure requires reprogramming of the PM immediately before and after the MR examination in the MR unit to keep the duration of the deactivation of the PM function as short as possible. Moreover, short-term - possibly emergency - reprogramming of the PM to its original mode in the MR unit must be ensured to treat possibly occurring symptomatic bradycardic episodes.

2. Reprogramming to VVI/DDI mode

The advantage of this option is that the initial (pre-MRI) reprogramming of the PM can be performed at a different time with respect to the MR exam and at a different location outside the MR unit (e. g. the cardiology/PM outpatient clinic) since the VVI mode offers sufficient protection against spontaneous bradycardic episodes. Reprogramming up to a maximum of 24 hours before MR examination is recommended. Reprogramming with restoration of the original mode and a check of PM functionality and pacing capture thresholds must be performed immediately after MR imaging.

A disadvantage of this procedure is that the following two scenarios can occur: Despite programmed VVI mode, asynchronous stimulations during MR examination can be triggered 1 . by activation of the magnetic reed switch in the static magnetic field or 2 . by temporary switching to interference mode. The risk of these asynchronous stimulations triggering ventricular tachycardia or ventricular fibrillation in a "normal" PM patient without an arrhythmogenic myocardial substrate is very low from an electrophysiological standpoint but cannot be definitively ruled out. This underscores the necessity of qualified monitoring during MR examination with the emergency option of immediate defibrillation by the attending physician or an on-call emergency team.

c) The stimulation pulse should be increased for all PM/ICD patients to four times the pacing capture threshold or to 5.0 volts/1.0 ms to compensate for a possibly occurring increase in pacing capture thresholds due to RF-induced heating of the PM leads.

d) The sensing and stimulation polarity of the PM leads should be reprogrammed to bipolar (if possible).

e) Additional stimulation functions (e. g. frequency-adapted stimulation, antitachycardia pacing) must be deactivated.

f) The time at which the PM is reprogrammed in relation to the MR examination (immediately prior to MR examination vs. at a different time and location than the MR examination) depends on the electrophysiological status of the patient ( $\vee$ Table 3 ). In every case the PM should be reprogrammed immediately after MR examination to detect possible MR-induced alterations of the PM system (e.g. electrical reset, pacing capture threshold increase, battery depletion) and to restore the original mode optimized to the individual patient status.

g) Follow-up to rule out long-term damage (e. g. chronic pacing capture threshold increase due to the formation of scar tissue) should be performed after 3 months.

\section{Monitoring in the case of conventional pacemakers}

A decisive safety element in the procedural management of patients with conventional pacemakers is adequate monitoring of vital functions in order to detect and treat potentially life-threatening complications early - in particular the occurrence of tachyand bradyarrhythmias.

Ventricular tachyarrhythmias can occur during an MR examination in PM patients:

1. as a result of asynchronous stimulations due to the abovedescribed interactions of the PM with the MR system and

2. as a result of voltage pulses induced in the leads with consecutive myocardial stimulation in the case of coupling of the electromagnetic MR fields into the PM lead loop

Bradyarrhythmias during an MR examination can occur:

1. in patients without PM dependency with intended deactivation of the stimulation function (0D0, 000; see above) as a result of spontaneous temporary episodes of bradycardia due to the primary disease of the patient,

2. in patients with absolute PM dependency with programming to the D00 mode as a result of MR-related electrical reset to the VVI mode and inhibition of the stimulation function by the pulsed MR fields,

3. in patients with absolute PM dependency due to ineffective stimulation in the case of an increase of the pacing capture thresholds as a result of thermal damage in the region of the lead tip.

Continuous pulse oximetry monitoring displaying the peripheral pulse wave and oxygen saturation using an MR conditional pulse 
- Table 3 Recommendations regarding reprogramming and monitoring in patients with conventional pacemakers undergoing MR imaging.

\begin{tabular}{|c|c|c|c|c|}
\hline patient status & MRI mode & reprogramming pre-MRI & monitoring during MRI & reprogramming post-MRI \\
\hline absolute PM dependency & D00, V00 & $\begin{array}{l}\text { possible at a different time and } \\
\text { location than MR examination } \\
\text { but on the same day as MRI }\end{array}$ & $\begin{array}{l}\text { presence of a cardiolo- } \\
\text { gist required }\end{array}$ & immediately post-MRI \\
\hline $\begin{array}{l}\text { no PM dependency; } \\
\text { stable/permanent } \\
\text { bradycardia RF }\end{array}$ & D00, V00 & $\begin{array}{l}\text { possible at a different time and } \\
\text { location than MR examination } \\
\text { but on the same day as MRI }\end{array}$ & $\begin{array}{l}\text { qualified medical per- } \\
\text { sonnel present; cardiolo- } \\
\text { gist available on an } \\
\text { emergency standby basis }\end{array}$ & immediately post-MRI \\
\hline $\begin{array}{l}\text { no PM dependency; } \\
\text { no stable/permanent } \\
\text { bradycardia } \\
\text { Option A }\end{array}$ & 0D0, 000 & immediately pre-MRI & $\begin{array}{l}\text { qualified medical per- } \\
\text { sonnel present; cardiolo- } \\
\text { gist available on an } \\
\text { emergency standby basis }\end{array}$ & immediately post-MRI \\
\hline $\begin{array}{l}\text { no PM dependency; } \\
\text { no stable/permanent } \\
\text { bradycardia } \\
\text { Option B }\end{array}$ & VVI, DDI & $\begin{array}{l}\text { possible at a different time and } \\
\text { location than MR examination } \\
\text { but within } 48 \text { hours before MRI }\end{array}$ & $\begin{array}{l}\text { qualified medical per- } \\
\text { sonnel present; cardiolo- } \\
\text { gist available on an } \\
\text { emergency standby basis }\end{array}$ & immediately post-MRI \\
\hline
\end{tabular}

oximeter certified for patient monitoring in the MR environment is mandatory. Moreover, additional ECG monitoring via a certified and MR-conditional ECG unit should be performed. However, it must be taken into consideration that, even in these dedicated devices, ECG registrations during active MR imaging often have significant overlaying artifacts so that diagnostic information can often only be obtained in MR imaging pauses.

On-site availability of an external defibrillator must be ensured. Moreover, a PM programmer must be available on-site or on an emergency basis. It should be noted that all emergency measures for patients requiring the use of a defibrillator or programmer or other emergency equipment with ferromagnetic components must be performed outside the MR examination room since a strong attractive force is exerted on these devices in the vicinity of the MR system thus representing an immediate danger for patients and medical staff.

In constellations with a higher risk profile ( $\bullet$ Table 1$)$ - primarily in the case of absolute PM dependency, abandoned PM leads, additional leads in CRT systems and in the case of a myocardium with a potentially arrhythmogenic substrate (e.g. subacute myocardial infarction, acute myocarditis) - a cardiologist must be present. The reason for the presence of a cardiologist is the need for immediate proper treatment in the case of clinically relevant episodes of bradyarrhythmia and ventricular tachyarrhythmia and the need of immediate treatment according to guidelines.

Abandoned PM leads are specified in the ESC guidelines from 2013 [21] as an absolute contraindication for an MR examination. From the viewpoint of the authors of this consensus paper, abandoned leads - as specified above - should be considered to significantly increase the risk profile. However, an MR examination can be performed in justified individual cases with clinical urgency and under consideration of the individual risk/benefit ratio. This applies in particular when the MR examination does not involve the thoracic region and when the patient does not have absolute
PM dependency so that the main risk of abandoned leads, namely RF-induced heating with an increase of the pacing capture threshold and consecutive stimulation loss, becomes less relevant.

In the case of "uncomplicated" constellations with a low risk profile (patient without PM dependency, no MR examination in the thoracic region, no abandoned leads or other risk-increasing parameters), monitoring can be performed on-site by qualified medical personnel provided that a cardiologist is available on an emergency standby basis and proper initial care of the patient can be ensured until the arrival of a cardiologist.

\section{MR-related safety precautions for conventional pacemakers}

By far, the most numerous clinical experiences are with closed/ cylindrical MR units with a field strength of 1.5T; thus these MR systems are preferable for scheduled MR examinations of patients with conventional pacemakers [3, 13, 14, 16, 23 -26]. There are studies demonstrating the safety of MR imaging of the brain at a field strength of $3 \mathrm{~T}$ using specific safety precautions [12]. However, it must be noted that doubling of the resonance frequency from appr. $64 \mathrm{MHz}$ (1.5 Tesla) to $128 \mathrm{MHz}$ (3.0 Tesla) increases the RF energy by a factor of four and thus the permissible limit values of the specific absorption rate (SAR value) using identical sequence parameters are reached sooner. Outside the head region, it is thus significantly more difficult to control and limit SAR values and RF-induced heating effects at 3 Tesla.

Open MR systems should not be used due to a lack of data.

The integrated RF body coil should be used as the RF transmit coil. Local transmit coils are absolutely contraindicated in the thoracic region. The use of local receive coils is non-critical and possible in all regions of the body - including the chest.

RF-induced heating of the PM leads cannot be predicted in individual cases, as it is dependent on numerous influencing parameters, including the position and configuration of the PM leads in the patient as well as their position relative to the 
RF transmitter coil, relation of the wave length of the RF excitation pulse to the effective lead length, and SAR value (specific absorption rate) of the MR sequence used. The SAR value (unit W/kg) is a measure of the absorption of electromagnetic field energy in biological tissue. To limit consecutive tissue heating during an MR scan, the radio frequency power irradiated into the body is monitored by the MR system and the corresponding SAR value is displayed. In keeping with legally mandated IEC threshold values, the SAR value during an MR examination must not exceed $4 \mathrm{~W} / \mathrm{kg}$ in the whole body or $3.2 \mathrm{~W} / \mathrm{kg}$ in the head independent of the presence or absence of a pacemaker or an otherwise active or passive implant.

If all other influencing parameters remain constant, there is a linear relationship between the SAR value of the related MR sequence and tissue heating at the tip of the pacemaker lead. This RF-induced tissue heating can thus be simply and effectively reduced by limiting the SAR value.

It is therefore strongly recommended that the whole body SAR value of all MR sequences should be limited to $<2 \mathrm{~W} / \mathrm{kg}$ (upper threshold of normal operating mode) during MR examinations of patients with conventional pacemakers.

Note: While there are currently clearly defined limits regarding the level of radio frequency power irradiated into the body and the gradient strengths to be used, there is a need for specification and optimization regarding the measurement techniques and measurement accuracy employed by individual MRI system manufacturers to monitor these limits and ensure that they are met.

\section{MR imaging of patients with MR conditional pacemaker systems}

MR conditional pacemakers have been tested for a MR examination under specific conditions and approved for use by the European Medical Device Directive with CE certification ("in-label” use). Pacemaker manufacturers guarantee safety with proper use according to the specific conditions for use.

Technical modifications of MR conditional pacemaker systems include replacement of the reed switch with a Hall sensor (unlike a standard reed switch, its behavior is predictable in a strong static magnetic field), improved protection of the internal circuits (thereby avoiding electromagnetic interference and voltage drop-induced electrical resets), modification of input capacities and protective diodes in the pacemaker pulse generator (to reduce voltage induction into the leads), as well as a software-based MR protection mode (see below).

Most pacemaker manufacturers have developed new lead models in which RF-induced heating is significantly reduced by specific design modifications (e. g. implementation of an electronic filter behind the lead tip). In addition, all major manufacturers have tested their conventional pacemaker leads and have identified numerous models, which under certain conditions could be approved as MR conditional (back labeling). This implies that even a conventional lead primarily labeled "MR-unsafe" on the pacemaker ID card issued at the time of implantation can be retrospectively tested and approved as "MR conditional". This emphasizes that data regarding MR safety of the leads in the pacemaker
ID card may not be reliable, and should be currently reviewed with the manufacturer (manual, hotline, website).

The recommended procedural management of patients with MR conditional pacemakers is shown in the following. It should be noted that all information is only a snapshot of the current situation. Changes to the conditions for use are possible, and it is necessary to review each case individually and in a timely manner.

\section{Indications/review of the conditions of use in the case of MR conditional pacemaker systems}

In the cardiac/pacemaker outpatient clinic PM interrogation and, if necessary, a review of the medical record of the center performing the PM implantation and in cases of doubt an X-ray of the chest are performed to determine whether the pacemaker-specific conditions of use for MR imaging are fulfilled:

1. Verification of a complete and approved MR conditional pacemaker system consisting of an MR conditional pacemaker generator and MR conditional pacemaker leads

2. Left or right pectoral implantation site of the pacemaker system

3. Implantation time $>6$ weeks

4. Electrically intact pacemaker leads

5. Pacing capture thresholds within the normal range

6. Sufficient battery capacity based on the manufacturer's specification

7. No additional cardiac leads (particularly no abandoned pacemaker leads), no additional lead components such as lead adapters or lead extensions

8. Exclusion of other cardiac implants, depending on the PM manufacturer's specification

9. Written documentation (checklist) by the attending cardiologist indicating that the electrophysiological conditions of use of the pacemaker have been fulfilled

Radiology (in the case of cardiac MR examinations, possibly the responsible cardiology department) performs verification of the MR-related conditions of use regarding

1. Design and field strength of the MR system

2. Strength of the gradient system regarding amplitude and slew rate

3. Whole-body vs. partial-body approval of the PM system

4. Absence of other extracardiac implants that rule out MR imaging, depending on the PM manufacturer's specification

5. Final written documentation (checklist) by the attending responsible experienced physician indicating that the MRrelated conditions of use of the pacemaker have been fulfilled.

\section{Patient information/informed consent in the case of MR conditional pacemaker systems}

It is recommended to inform the patient that MR conditional pacemaker systems are new technology for which only limited long-term clinical data is currently available. MR-associated risks such as lead heating and/or unintended cardiac stimulation are minimized to the extent that the remaining risk is accepted as reasonable according to the criteria of the approval authorities under consideration of the benefits to be expected from the 
MR examination. However, these risks cannot be absolutely ruled out in individual cases.

\section{Monitoring during MR examinations in the case of MR conditional pacemaker systems}

In principle, the presence of a cardiologist during an MR scan of a patient with an MR conditional pacemaker system is not required as long as complications which may occur independent of the presence of a pacemaker can be detected and treated by attending medical staff until the arrival of an emergency team. The situation is comparable to the management of contrast agentinduced events.

All manufacturers of MR conditional pacemakers require patient monitoring using the following methods during an MR scan: pulse oximetry, ECG or blood pressure measurement. The user may select the monitoring modality. The authors of this consensus paper strongly recommend monitoring using an MR conditional pulse oximeter since the validity of ECG registrations is still frequently impaired by significant overlaying artifacts and blood pressure measurements do not provide continuous monitoring of the patient. In contrast to the procedure for conventional pacemakers, pulse oximetry alone is considered sufficient for monitoring patients with MR conditional pacemaker systems.

\section{MR-related conditions for use/safety precautions for MR conditional pacemaker systems}

SAR value: To reduce RF-induced lead heating, the whole-body SAR value is limited for most MR conditional systems to the normal operating mode with an upper threshold of $2 \mathrm{~W} / \mathrm{kg}$ (local head SAR value $3.2 \mathrm{~W} / \mathrm{kg}$ ) (see also the comment "SAR value" in the section "Conventional pacemakers"). Some pacemaker systems made by Biotronik, Boston Scientific and St. Jude are also approved for MR examinations with a maximum possible wholebody SAR value of $4 \mathrm{~W} / \mathrm{kg}$.

MR coils: All currently commercially available receiver coils, including local surface receiver coils, can be used in all body regions. The integrated body RF coil is approved for all pacemaker manufacturers as the transmitter coil. Some pacemaker manufacturers (Medtronic, Biotronik and Boston Scientific) also allow the use of combined transmit/receive coils outside the thoracic region which in relatively rare cases are available for some MR systems as knee or head coils, for example.

There are additional manufacturer and model-dependent specifications of the conditions of use for MR conditional pacemaker systems, such as MR examination time, cumulative MR examination time over the life time of the pacemaker system, minimum patient body size, position of the patient within the RF coil, and exclusion of fever or impaired patient body temperature regulation, which will not be discussed here in greater detail. The position paper of the German Roentgen Society regarding MR examinations in patients with pacemakers provides a detailed overview of these conditions for use [22]. However, it is the responsibility of the attending radiologist to obtain the conditions for use that are valid at the time of MR examination from the product manuals, hotlines or website of the relevant pacemaker manufacturer.

\section{Pacemaker-related conditions for use/safety precautions for MR conditional pacemaker systems}

Analogously to the procedure with conventional pacemaker systems, prior to MR imaging, MR conditional PM systems should be programmed to an MR protection mode (asynchronous mode or deactivation of the pacing function, bipolar lead configuration, increased stimulation output, deactivation of supplementary stimulation functions such as frequency-adapted stimulation or antitachycardia overstimulation), which is stored in the software options of the pacemaker model. This implies that patients must always and principally be seen by a cardiologist before and after an MR examination.

Recommendations regarding reprogramming and monitoring in MR conditional pacemakers are provided in - Table 4.

Unlike conventional pacemaker systems, follow-up after 3 months to rule out long-term damage is not obligatory.

\section{MR imaging in patients with conventional ICD systems}

Compared to pacemakers, implantable cardioverter defibrillators (ICD) have additional electrotechnical components such as a transformer to increase the battery voltage and a capacitor for storing the energy needed for shock delivery.

A further fundamental design and safety-related difference is that magnetic activation of the reed switch does not result in asynchronous stimulation as in pacemakers but rather in deactivation of tachycardia detection and thus in deactivation of antitachycardia therapy (shock delivery and ventricular overstimulation for the termination of ventricular fibrillation and/or ventricular tachycardia).

All of the main risks for patients with pacemakers (including inhibition of pacemaker stimulation, unintended cardiac stimulation, heating of leads) also apply to ICD patients.

\section{However, the risks posed by MR imaging are significantly higher in ICD patients:}

1. In contrast to pacemaker patients, ICD patients typically have a structural myocardial disease. As a result, there is a comparably higher risk of triggering ventricular tachycardia or ventricular fibrillation in the case of voltage inductions at the lead tips occurring during MR imaging.

2. Additional complex interactions between the ICD and the specific MR fields are the result of the additional diagnostic and therapeutic antitachycardia functions. Pulsed MR fields can be interpreted by the ICD system as ventricular tachycardia or ventricular fibrillation and thus may induce therapeutic shock delivery. In principle, this problem can be avoided by deactivation of ICD treatment options prior to MR examination. However, it must be taken into consideration that sensing of the MR fields as electromagnetic interference can cause the ICD system to perform an electrical reset in which the therapy option of the ICD system is activated/reactivated by default. However, the potential risk for the patient here is not inappropriate shock delivery by the ICD system as would be ex- 
pected, since the ferromagnetic core of the transformer is saturated in the strong static magnetic field of an MR system which makes charging of the ICD capacitors impossible. Instead, the main risk for patients is that the short-circuit of the battery in the static magnetic field cause repetitive unsuccessful charging attempts which may result in heating and damage of the electronic components of the ICD, leading to complete and permanent system failure. This would result in an acute and potentially life-threatening emergency situation for ICD patients with absolute PM dependency. Moreover, the pulse generator can be significantly heated by the short-circuit resulting in local thermal damage.

Note: Some ICD models allow programming of audio warning sounds to indicate, e.g. battery depletion or electrode dysfunction. These alert features may fail after MR imaging due to damage to the ICD loudspeaker unit.

\section{Critical review of the indications for MR imaging in patients with conventional ICD systems}

Indications should be determined and reviewed as in the case of conventional pacemakers.

It is essential to evaluate the individual risk/benefit ratio for the patient. The particular region to undergo MR examination and the electrophysiological risk profile of the ICD patient in particular must be taken into consideration when determining risk.

It must be stated again here that the electrophysiological risk for an ICD patient during MR examination is to be considered significantly higher compared to PM patients for the following reasons: 1 . the higher vulnerability of the structurally damaged myocardium and 2. the higher risk of irreversible malfunctioning of the ICD system.

Particularly in the case of a very high or high risk profile ( $\vee$ Table 5 ), the indication for an MR examination should be determined on a very strict basis and the expected risk/benefit ratio should be critically reviewed.

\section{Patient education/informed consent in patients with conventional ICD systems}

1. As in the case of conventional pacemaker systems, MR imgaging in patients with conventional ICD systems is a nonapproved ("off-label") procedure based on a case-by-case decision on the part of the attending responsible expert physicians.

2. MR imgaging in patients with conventional ICD systems pose the following potential risks and complications:

- Damage to the ICD system to the point of complete system failure as a potentially life-threatening complication in patients with absolute PM dependency

- Malfunction of the leads, e. g. due to heating-related increase of the pacing capture threshold, with the need for lead revision/extraction

- Heating of the leads resulting in thermal myocardial/subendocardial damage and ineffective stimulation (acute or chronic) as a potentially life-threatening complication in the case of absolute PM dependency of the patient

- Induction of potentially life-threatening tachyarrhythmias
- Inhibition of pacemaker therapy of spontaneous bradyarrhythmic episodes occurring during the MR scan with potentially life-threatening consequences in patients with absolute PM dependency

- Due to the necessary deactivation of the ICD therapy options, the patient is not protected in the case of spontaneous potentially life-threatening tachyarrhythmias occurring during the MR examination

The risks mentioned above can be minimized by employing specific safety precautions. However, these risks cannot be absolutely eliminated and are not quantifiable in individual cases.

\section{Reprogramming strategies in patients with conventional ICD systems}

In particular, it must be taken into consideration in reprogramming strategies for patients with conventional ICD systems that the occurrence of intermittent asynchronous myocardial stimulation and competitive rhythms should be strictly avoided taking into account the increased risk of the induction of high-grade ventricular arrhythmias due to the structurally damaged myocardium of ICD patients.

Consequently, ICD patients with stable intrinsic bradycardia should be programmed to D00 or V00 mode with asynchronous stimulation significantly above the intrinsic heart rate (e. g. 80 or $90 / \mathrm{min})$. This programming also takes into consideration that hemodynamically limited ICD patients typically do not tolerate a low intrinsic heart rate well. If the conventional ICD system does not support asynchronous stimulation in bradycardic ICD patients, the indication for MR imaging should be determined on a stricter basis due to the risk of complete inhibition of ICD stimulation in DDD or VVI mode (see above).

MR imaging in ICD patients with absolute PM dependency represents a particularly high risk due to the danger of MR induced complete system failure (see above) and should only be performed in justified exceptional cases at highly experienced centers.

In ICD patients who are not PM-dependent, the ICD should be programmed to 0D0 mode with deactivation of the stimulation function.

It is absolutely mandatory to deactivate tachycardia detection prior to the MR examination to avoid misinterpretation of the pulsed MR fields as malignant ventricular arrhythmias and attempts of the ICD to deliver shock therapy.

As in the case of conventional pacemakers, an ICD follow-up to rule out long-term damage should be performed after 3 months.

This recommended reprogramming procedure is summarized in Table 5.

\section{Monitoring in patients with conventional ICD systems}

As in the case of pacemaker patients, MR-compatible pulse oximetry with continuous display of the peripheral pulse wave and oxygen saturation is mandatory for the monitoring of ICD patients. Additional ECG monitoring with a certified and MR conditionals ECG device is recommended.

Moreover, on-site availability of a defibrillator and a suitable ICD programmer is absolutely mandatory. 
- Table 4 Recommendations regarding reprogramming and monitoring in patients with MR conditional pacemakers undergoing MRI.

\begin{tabular}{|c|c|c|c|c|}
\hline patient status & MRI mode & reprogramming pre-MRI & monitoring during MRI & reprogramming post-MRI \\
\hline absolute PM dependency & D00, V00 & $\begin{array}{l}\text { possible at a different time and } \\
\text { location than MR examination but } \\
\text { on the same day as MRI }\end{array}$ & $\begin{array}{l}\text { qualified medical person- } \\
\text { nel present; cardiologist } \\
\text { available on an emergen- } \\
\text { cy standby basis }\end{array}$ & $\begin{array}{l}\text { possible at a different time and } \\
\text { location than MR examination } \\
\text { but on the same day as MRI }\end{array}$ \\
\hline $\begin{array}{l}\text { no PM dependency; } \\
\text { stable/permanent } \\
\text { bradycardia }\end{array}$ & D00, V00 & $\begin{array}{l}\text { possible at a different time and } \\
\text { location than MR examination but } \\
\text { on the same day as MRI }\end{array}$ & $\begin{array}{l}\text { qualified medical person- } \\
\text { nel present; cardiologist } \\
\text { available on an emergen- } \\
\text { cy standby basis }\end{array}$ & $\begin{array}{l}\text { possible at a different time and } \\
\text { location than MR examination } \\
\text { but on the same day as MRI }\end{array}$ \\
\hline $\begin{array}{l}\text { no PM dependency; } \\
\text { no stable/permanent bradycardia } \\
\text { Option } \mathrm{A}^{1}\end{array}$ & $0 \mathrm{D} 0,000^{1}$ & immediately pre-MRI & $\begin{array}{l}\text { qualified medical person- } \\
\text { nel present; cardiologist } \\
\text { available on an emergen- } \\
\text { cy standby basis }\end{array}$ & immediately post-MRI \\
\hline $\begin{array}{l}\text { no PM dependency; } \\
\text { no stable/permanent bradycardia } \\
\text { Option } \mathrm{B}^{2}\end{array}$ & $\mathrm{VVI}^{2}$ & $\begin{array}{l}\text { possible at a different time and } \\
\text { location than MR examination but } \\
\text { within } 48 \text { hours before MRI }\end{array}$ & $\begin{array}{l}\text { qualified medical person- } \\
\text { nel present; cardiologist } \\
\text { available on an emergen- } \\
\text { cy standby basis }\end{array}$ & $\begin{array}{l}\text { possible at a different time and } \\
\text { location than MR examination } \\
\text { but within } 48 \text { hours after MRI }\end{array}$ \\
\hline \multicolumn{5}{|c|}{$\begin{array}{l}1 \text { Note: } 0 \mathrm{D} 0 \text { or } 000 \text { mode is recommended by manufacturers of MR conditional pacemakers due to the minimization of possible interference with the MR } \\
\text { fields and is stored in the MR protection mode. However, in OD0/000 mode, the patient is not protected during MR imaging against spontaneous sympto- } \\
\text { matic bradycardia. Therefore, appropriate emergency cardiology care must be ensured. } \\
2 \text { Note: VVI mode allows reprogramming at a different time and location than the MR examination but entails a higher risk for interference with MR fields and } \\
\text { is therefore not part of the stored MR protection mode, thus representing off-label use. }\end{array}$} \\
\hline
\end{tabular}

Since ICD treatment options are deactivated for MR examination and the patient is thus not protected in the case of spontaneous high-grade arrhythmias, the patient must be continuously monitored until the original ICD mode is restored.

Reprogramming should be performed immediately before and after MR examination. The presence of a cardiologist is mandatory since any complications that occur (ventricular fibrillation, ineffective stimulation in a patient with PM dependency) in ICD patients with a structurally damaged myocardium require quick and specific electrophysiological management.

\section{MR-related safety precautions in patients with conventional ICD systems}

The following MR-related safety precautions for patients with conventional ICD systems should be used analogously to the procedure for patients with conventional pacemakers:

- Restriction to closed/cylindrical MR units with a field strength of 1.5 Tesla

- Integrated body RF coil as an RF transmitter coil. Local receiver coils are non-critical in all regions of the body

- Limitations of the whole-body SAR value of all MR sequences to $<2 \mathrm{~W} / \mathrm{kg}$ (upper threshold of the normal operating mode) or to $<3.2 \mathrm{~W} / \mathrm{kg}$ in the head

- Avoidance of MR pulse sequences with steep and fast gradients if technically possible by modifying the sequence parameter to a lower gradient output

- Limitation of the active MR examination time to 30 min

\section{MR imaging of patients with} MR conditional ICD systems

MR conditional ICD systems have been tested in the MR environment and approved for use under specific conditions by the European Regulatory Authorities with CE certification ("in-label” use).

The specific conditions for use correspond largely to those of MR conditional PM systems and must be obtained from the current manual, hotline, or website of the particular manufacturer.

Recommendations regarding risk assessment, reprogramming and monitoring in MR examinations of patients with conventional and MR conditional ICD systems are provided in \ Table 5.

\section{Cardiac MR imaging of patients with PM and ICD systems}

The recommendations regarding risk assessment, reprogramming, and monitoring of patients with pacemakers and ICDs undergoing cardiac MR ( $\vee$ Table 6 ) fundamentally correspond to the recommendations given above for PM/ICD patients undergoing extracardiac MR.

However, cardiac MR imaging principally poses a higher risk for the following reasons:

1. Higher risk profile due to the position of the PM/lead system in the central region of the RF coil during cardiac MR examination with full irradiation of the RF energy

2. Higher risk of relevant impairment of the image quality of cardiac MR examinations due to susceptibility and off-reso- 
- Table 5 Recommendations regarding risk assessment, reprogramming and monitoring in patients with conventional and MR conditional ICD systems undergoing MR imaging.

\begin{tabular}{|c|c|c|c|c|}
\hline risk & MRI mode ${ }^{1}$ & reprogramming pre-MRI & monitoring during MRI & reprogramming post-MRI \\
\hline $\begin{array}{l}\text { very high } \\
\text { (conventional ICD plus absolute pace- } \\
\text { maker dependency) }\end{array}$ & D00 & immediately before MR & $\begin{array}{l}\text { presence of a cardiologist } \\
\text { required }\end{array}$ & immediately after MRI \\
\hline $\begin{array}{l}\text { high } \\
\text { (conventional ICD plus history of VT/VF, } \\
\text { no pacemaker dependency) }\end{array}$ & OD0 or $\mathrm{D}^{2} 0^{2}$ & immediately before MRI & $\begin{array}{l}\text { presence of a cardiologist } \\
\text { required }\end{array}$ & immediately after MRI \\
\hline $\begin{array}{l}\text { medium } \\
\text { (conventional ICD, no history of VT/VF, } \\
\text { no pacemaker dependency) }\end{array}$ & OD0 or $\mathrm{D} 00^{2}$ & immediately before MRI & $\begin{array}{l}\text { presence of a cardiologist } \\
\text { required }\end{array}$ & immediately after MRI \\
\hline $\begin{array}{l}\text { low } \\
\text { (MR conditional ICD plus absolute } \\
\text { pacemaker dependency) }\end{array}$ & D00 & immediately before MRI & $\begin{array}{l}\text { presence of a cardiologist } \\
\text { required }\end{array}$ & immediately after MRI \\
\hline $\begin{array}{l}\text { very low } \\
\text { (MR conditional ICD, no history of VT/ } \\
\text { VF, no pacemaker dependency) }\end{array}$ & OD0 or $\mathrm{D} 00^{2}$ & immediately before MRI & $\begin{array}{l}\text { qualified medical personnel } \\
\text { present; cardiologist avail- } \\
\text { able on an emergency stand- } \\
\text { by basis }\end{array}$ & immediately after MRI \\
\hline
\end{tabular}

- Table 6 Recommendations regarding reprogramming and monitoringin patients with conventional pacemakers undergoing cardiac MRI.

\begin{tabular}{|l|l|l|l|l|}
\hline patient status & MRI mode & reprogramming pre-MRI & monitoring during MRI & reprogramming post-MRI \\
\hline absolute PM dependency & D00, V00 & $\begin{array}{l}\text { possible at a different time and loca- } \\
\text { tion than MR examination but on the } \\
\text { same day as MRI }\end{array}$ & $\begin{array}{l}\text { presence of a cardiologist } \\
\text { required }\end{array}$ & $\begin{array}{l}\text { immediately } \\
\text { post-MRI }\end{array}$ \\
\hline $\begin{array}{l}\text { no PM dependency; } \\
\text { stable/permanent bradycardia }\end{array}$ & D00, V00 & $\begin{array}{l}\text { possible at a different time and loca- } \\
\text { tion than MR examination but on the } \\
\text { same day as MRI }\end{array}$ & $\begin{array}{l}\text { presence of a cardiologist } \\
\text { required }\end{array}$ & immediately \\
post-MRI
\end{tabular}

nance artifacts that arise particularly from the transformer in ICD systems and require the use of alternative sequence techniques (e. g. spoiled gradient sequences instead of SSFP sequences, broadband excitation pulses in late enhancement sequences)

3. Higher risk of competitive rhythms and induction of cardiac arrhythmias during cardiac MR stress tests due to unstable/ stimulated cardiac heart rates

4. The indication for cardiac MR examinations usually implies the presence or suspicion of structural myocardial disease with a higher risk profile (e. g. myocarditis, cardiomyopathy) for MR imaging.

Therefore, the indication for cardiac MR examinations in PM/ICD patients should be determined on a particularly strict basis, especially if the issue can be adequately resolved with alternative diagnostic methods such as echocardiography or SPECT and MR imaging should only be performed at highly experienced centers. 


\section{Summary:}

1. Conventional PM and ICD systems are no longer an absolute but rather a relative contraindication for performing an MR examination. Procedural management includes the assessment of the individual risk/benefit ratio, comprehensive patient informed consent about specific risks and "off label" use, extensive PM/ICD-related and MR-related safety precautions to reduce these risks to the greatest extent possible, as well as adequate monitoring techniques.

2. MR conditional pacemaker and ICD systems have been tested and approved for MR examination under specific conditions ("in-label” use). Precise understanding of and compliance with the terms of use for the specific pacemaker system are essential for patient safety.

3. The risk for an ICD patient during MR examinations is to be considered significantly higher compared to PM patients due to the higher vulnerability of the structurally damaged myocardium and the higher risk of irreversible damage to conventional ICD systems. The indication for an MR examination of an ICD patient should therefore be determined on a stricter basis and the expected risk/benefit ratio should be critically reviewed.

4. Cardiac MR represents a special case requiring stricter indications both for conventional and MR conditional PM and ICD systems due to the particular higher risk profile. Specific procedural recommendations are being developed by the corresponding cardiology and radiology societies.

5. This complex subject requires close collaboration between radiology and cardiology.

\section{Conflicts of Interest}

\section{T. Sommer: Consultant: Medtronic, Biotronik, Boston Scientic} W. Bauer: Research grant/clinical studies: DFG, BMBF, ZIM-Wirtschaftsministerium, Philips, Siemens, General Electric; Scientific advisor: Biotronik; Honorarium (lectures): Pfizer, Biotronik, BerlinPharma, Sanofi, Bayer

C. Kolb: Honorarium (lectures)/travel support: Biotronik, Boston Scientific, LivaNova/Sorin Group, Medtronic, Spectranetics, St. Jude; Medical-Consultant: Biotronik, Boston Scientific, LivaNova/ Sorin Group; Clinical Study Support: Biotronik, Boston Scientific, LivaNova/Sorin Group, Medtronic, St. Jude Medical.

R. Luechinger: Consultant: Medtronic

U. Wiegand: Honorarium (lectures): Medtronic, St. Jude Medical

M. Keim: Consultant: Miracor Medical Systems; Honorarium (lectures and trainings): Bayer; Research grant/clinical studies: Medtronic, Edwards, Philips, third-party founds.

D. Bäntsch: Consultant: Biotronik; Honorarium (lectures): Biotronic, Boston Scientific, Medtronic, St. Jude Medical, Research grants: Biotronik, Medtronic

\section{References}

[1] Roguin A, Schwitter ], Vahlhaus C et al. Magnetic resonance imaging in individuals with cardiovascular implantable electronic devices. Europace 2008; 10: $336-346$
[2] Sommer T, Naehle CP, Yang A et al. Strategy for safe performance of extrathoracic magnetic resonance imaging at 1.5 tesla in the presence of cardiac pacemakers in non-pacemaker-dependent patients: a prospective study with 115 examinations. Circulation 2006; 114: 1285 1292

[3] Nazarian S, Hansford R, Roguin A et al. A prospective evaluation of a protocol for magnetic resonance imaging of patients with implanted cardiac devices. Annals of internal medicine 2011; 155: 415-424

[4] Nazarian S, Halperin HR. How to perform magnetic resonance imaging on patients with implantable cardiac arrhythmia devices. Heart Rhythm 2009; 6: $138-143$

[5] Roguin A, Zviman MM, Meininger GR et al. Modern pacemaker and implantable cardioverter/defibrillator systems can be magnetic resonance imaging safe: in vitro and in vivo assessment of safety and function at 1.5 T. Circulation 2004; 110: 475-482

[6] Nazarian S, Beinart R, Halperin HR. Magnetic resonance imaging and implantable devices. Circulation Arrhythmia and electrophysiology 2013; 6: 419-428

[7] Sommer T, Vahlhaus C, Lauck G et al. MR imaging and cardiac pacemakers: in-vitro evaluation and in-vivo studies in 51 patients at $0.5 \mathrm{~T}$. Radiology 2000; 215: 869 -879

[8] Luechinger R, Duru F, Scheidegger MB et al. Force and torque effects of a 1.5-Tesla MRI scanner on cardiac pacemakers and ICDs. Pacing Clin Electrophysiol 2001; 24: 199-205

[9] Luechinger R, Duru F, Zeijlemaker VA et al. Pacemaker reed switch behavior in $0.5,1.5$, and 3.0 Tesla magnetic resonance imaging units: are reed switches always closed in strong magnetic fields? Pacing Clin Electrophysiol 2002; 25: 1419-1423

[10] Luechinger R, Zeijlemaker VA, Pedersen EM et al. In vivo heating of pacemaker leads during magnetic resonance imaging. Eur Heart J 2005; 26: $376-383$

[11] Tandri H, Zviman MM, Wedan SR et al. Determinants of gradient fieldinduced current in a pacemaker lead system in a magnetic resonance imaging environment. Heart Rhythm 2008; 5: 462-468

[12] Naehle CP, Meyer C, Thomas D et al. Safety of brain 3-T MR imaging with transmit-receive head coil in patients with cardiac pacemakers: pilot prospective study with 51 examinations. Radiology 2008; 249: 991 1001

[13] Naehle CP, Zeijlemaker V, Thomas D et al. Evaluation of cumulative effects of MR imaging on pacemaker systems at 1.5 Tesla. Pacing Clin Electrophysiol 2009; 32: 1526-1535

[14] Schmiedel A, Hackenbroch M, Yang A et al. Magnetic resonance imaging of the brain in patients with cardiac pacemakers. Experimental and clinical investigations at 1.5 Tesla. Fortschr Röntgenstr 2005; 177: 731 - 744

[15] Strach K, Naehle CP, Muhlsteffen A et al. Low-field magnetic resonance imaging: increased safety for pacemaker patients? Europace 2010; 12: $952-960$

[16] Nazarian S, Roguin A, Zviman MM et al. Clinical utility and safety of a protocol for noncardiac and cardiac magnetic resonance imaging of patients with permanent pacemakers and implantable-cardioverter defibrillators at 1.5 tesla. Circulation 2006; 114: 1277 - 1284

[17] Martin ET, Coman JA, Shellock FG et al. Magnetic resonance imaging and cardiac pacemaker safety at 1.5-Tesla. J Am Coll Cardiol 2004; 43 : $1315-1324$

[18] Mollerus M, Albin G, Lipinski M et al. Cardiac biomarkers in patients with permanent pacemakers and implantable cardioverter-defibrillators undergoing an MRI scan. Pacing Clin Electrophysiol 2008; 31: 1241 - 1245

[19] Mollerus M, Albin G, Lipinski M et al. Magnetic resonance imaging of pacemakers and implantable cardioverter-defibrillators without specific absorption rate restrictions. Europace 2010; 12: 947 - 951

[20] Nordbeck P, Weiss I, Ehses P et al. Measuring RF-induced currents inside implants: Impact of device configuration on MRI safety of cardiac pacemaker leads. Magn Reson Med 2009; 61: 570-578 
[21] Brignole M, Auricchio A, Baron-Esquivias G et al. 2013 ESC guidelines on cardiac pacing and cardiac resynchronization therapy: the task force on cardiac pacing and resynchronization therapy of the European Society of Cardiology (ESC). Developed in collaboration with the European Heart Rhythm Association (EHRA). Europace 2013; 15: 1070-1118

[22] Sommer T, Luechinger R, Barkhausen J et al. German Roentgen Society Statement on MR Imaging of Patients with Cardiac Pacemakers. Fortschr Röntgenstr 2015; 187: 777-787

[23] Markewitz A. Annual Report 2011 of the German pacemaker and defibrillator register: Section pacemakers and AQUA-Institute for Applied Quality Improvement and Research in Health Care. Herzschrittmacherther Elektrophysiol 2013; 24: 249-274

[24] Raatikainen M], Arnar DO, Zeppenfeld K et al. Current trends in the use of cardiac implantable electronic devices and interventional electrophysiological procedures in the European Society of Cardiology member countries: 2015 report from the European Heart Rhythm Association. Europace 2015; 17: iv1 - iv72

[25] Levine GN, Gomes AS, Arai AE et al. Safety of magnetic resonance imaging in patients with cardiovascular devices: an American Heart Association scientific statement from the Committee on Diagnostic and Interventional Cardiac Catheterization, Council on Clinical Cardiology, and the Council on Cardiovascular Radiology and Intervention: endorsed by the American College of Cardiology Foundation, the North American Society for Cardiac Imaging, and the Society for Cardiovascular Magnetic Resonance. Circulation 2007; 116: 2878-2891
[26] Naehle CP, Strach K, Thomas D et al. Magnetic resonance imaging at 1.5$\mathrm{T}$ in patients with implantable cardioverter-defibrillators. J Am Coll Cardiol 2009; 54: 549-555

[27] Gimbel JR. Unexpected asystole during 3T magnetic resonance imaging of a pacemaker-dependent patient with a modern pacemaker. Europace 2009; 11: $1241-1242$

[28] Irnich W, Irnich B, Bartsch C et al. Do we need pacemakers resistant to magnetic resonance imaging? Europace 2005; 7: 353-365

[29] Wilkoff BL, Bello D, Taborsky M et al. Magnetic resonance imaging in patients with a pacemaker system designed for the magnetic resonance environment. Heart rhythm 2011; 8: 65-73

[30] Gold MR, Sommer T, Schwitter ] et al. Full-Body MRI in Patients with an implantable Cardioverter-Defibrillator: Primary Results of a Randomized Study. J AM Coll Cardiol 2015; 65: 2581-2588

[31] Awad K, Griffin J, Crawford TC et al. Clinically safety of the Iforia implantable cardioverter-defibrillator system in patients subjected to thoracic spine and cardiac $1.5 \mathrm{~T}$ magnetic resonance imaging scanning conditions. Heart Rhythm 2015; 12: 2155-2161

[32] Bailey WM, Mazur A, McCotter C et al. Clinical safety of the ProMRI pacemaker system in patients subjected to thoracic spine and cardiac 1.5 T magnetic resonance imaging conditions. Heart Rhythm 2016; 13 : $464-471$ 